\title{
I/Q Imbalance Calibration for Higher Self-Interference Cancellation Levels in Full-Duplex Wireless Transceivers
}

\author{
Ramez Askar*, Nidal Zarifeh*, Benjamin Schubert ${ }^{\dagger}$, Wilhelm Keusgen ${ }^{\dagger}$, and Thomas Kaiser* \\ *Institute of Digital Signal Processing, Faculty of Engineering, University of Duisburg-Essen \\ D-47057 Duisburg, Germany \\ ${ }^{\dagger}$ Dept. of Wireless Communications and Networks, Fraunhofer Heinrich Hertz Institute \\ D-10587 Berlin, Germany
}

\begin{abstract}
This paper investigates a Full-Duplex transceiver using an active cancellation technique where an additional transmit chain is employed to create the self-interference cancellation signal. Both transmitters and also the receiver are impaired by frequency-dependent $I / Q$ imbalance with different parameters. It is shown how an I/Q parameter deviation in these chains deteriorates the achievable self-interference suppression level. Three different types of pre-equalization units, with variable complexity degree and different implementation approach, are then introduced and investigated. These units correct the I/Q imbalance and retain the self-interference level to its value as in a perfectly I/Q matched full-duplex transceiver. They can even be used to fully calibrate the I/Q imbalance. Simulative results show that self-interference suppression levels of more than $71 \mathrm{~dB}$ can be achieved.
\end{abstract}

\section{INTRODUCTION}

The continuous increasing demand for higher data rates and the competition in providing ubiquitous communication services raises the necessity of proposing schemes that revolutionize the radio resources utilization especially by ensuring higher efficiency in the usage of spectrum. Traditional wireless systems which use Time Division Duplexing (TDD) or Frequency Division Duplexing (FDD) schemes are suffering from specific limitation in resource utilization as they are halfduplex systems, which means they cannot transmit and receive at the same time in the same frequency band, hence they cannot achieve the desired spectrum efficiency.

Recently, the major interest has become about the fullduplex systems which can transmit and receive at the same time in the same frequency band. This gives more efficiency and flexibility in spectrum usage. The main challenge toward full-duplex systems is the self-interference cancellation. This means how to suppress the signal that leaks from the transmitter chain into the receiver chain of the same device (Base Station or Small Cell), taking into consideration that the transmitted signal power is in the order of $80-120 \mathrm{~dB}$ higher than the desired received signal power in most of standard systems like WiFi or LTE. Many techniques are suggested for self-interference cancellation such as in [1][5]. The cancellation techniques vary over different domains, from antenna domain like conditional placement [2], [6], over analog RF domain like a noise cancellation circuit with an RF Balun [7] or an analog circuit board [4], to a complementary digital cancellation algorithm or even a hybrid $\mathrm{RF} /$ digital solutions as proposed in [8], [9]. More related literature to this paper topic; the authors in [10] have proposed a solelydigital baseband approach to cancel the self-interference where a sufficient RF self-interference suppression are assumed. A widely-linear signal processing has been utilized in [10] to cope with the I/Q imbalances that occur in the transceiver chains.

The active cancellation mechanism [9] requires a complete additional transmission chain, which is dedicated to cancel the self-interference signal. Regardless of how accurately the transmission chains are implemented, the original chain and the cancellation one will not be totally identical and they will be distinctly affected by the hardware impairments. One of these impairments is the I/Q imbalance. The discrepancies of the I/Q imbalance parameters between the two transmission chains, which cause imprecise self-interference signal generation, are a major source of poor self-interference suppression performance.

The remainder of this paper is organized as follows: Section II introduces the structure and the equivalent baseband model of a full-duplex wireless transceiver that is impaired by I/Q imbalance and incorporates the active self-interference cancellation mechanism. Advanced pre-equalization units which are able to handle the I/Q impairment are proposed in Section III. In Section IV, the pilot signal structure is specified, and the parameter estimation procedure is presented. Section V presents simulation results and evaluates the pre-equalization units' performances. Finally, the conclusions are drawn in Section VI.

Notations: $\mathfrak{R}\{\ldots\}$ and $\mathfrak{J}\{\ldots\}$ denote the real and the imaginary parts of the signal, respectively. The convolution operator is indicated by $*$. The superscript $(\cdot)^{*}$ denotes the complex conjugate. $\mathcal{F}$ and $\mathcal{F}^{-1}$ denote the Fourier transform and inverse Fourier transform, respectively.

\section{Full-Duplex Transceiver AND IMPAIRMENTS IMPACT}

The active cancellation mechanism substantially relies on the precision of the self-interference cancellation signal. Higher precision of the self-interference cancellation signal can assure a significant improvement in self-interference suppression, which is the right course toward practical implementation of full-duplex systems. Throughout this paper, the phase noise of the local oscillator (LO) [11] is omitted to ease the notation and focus on explaining the influence of the I/Q imbalance. 


\section{A. Transceiver structure impaired with I/Q imbalance}

The full-duplex transceiver embraces the self-interference active cancellation mechanism with an extra transmission chain, indicated here as the auxiliary chain. Such approach has been studied in the literature [8], [9]. Fig. 1 depicts the structure of the full-duplex transceiver. The structure illustrates the imperfections of the LO signals as the main source of the frequency-independent I/Q imbalance. The LO signals $l_{\text {chain,arm }}(t)$ of the transceiver mixers are assumed to be uncorrelated to simplify the derivation and without any loss of generality. Other sources of I/Q imbalance due to the baseband filters mismatches and differences in group delay between the I- and Q-arm, which have a frequency selective behavior, are also considered in this work.

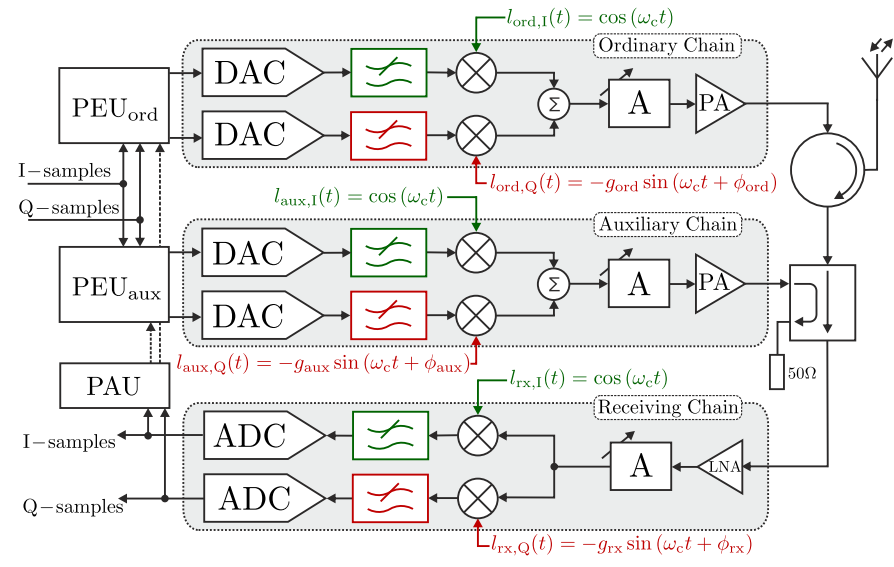

Fig. 1. Full-duplex transceiver structure. The block diagram indicates the drift in the local oscillator signals, $l_{\text {chain, } \operatorname{arm}}(t)$, from their nominal values.

The key enabling elements for a full-duplex scheme utilizing such transceiver structure are the digital pre-equalization units $\mathrm{PEU}_{\text {ord }}$ and $\mathrm{PEU}_{\mathrm{aux}}$ at the ordinary and auxiliary transmission chain, respectively. These digital blocks receive their parameters from a parameter acquisition unit (PAU) which is located at the receiving chain digital domain. Previous work [9] has utilized a basic linear model to equip the $\mathrm{PEU}_{\mathrm{aux}}$, while here in this work, advanced models of pre-equalization are developed to cope with transceiver imperfections, more specifically the I/Q imbalance.

\section{B. Signal and System Model}

The system model realizes the full-duplex transceiver structure with an active cancellation mechanism. As shown in Fig. 2, the equivalent baseband model of the $\mathrm{I} / \mathrm{Q}$ imbalance, such as in [12], is incorporated into the transceiver system model. This model is meant to study extensively the influence of the I/Q imbalance on the self-interference suppression performance.

$h_{\text {ord,I }}(t)$ and $h_{\text {ord,Q }}(t)$ represent the impulse response of the baseband filters in the ordinary transmission chain ${ }^{1}$ at the I- and Q-arm, respectively. $h_{\mathrm{SI}}(t)$ represents the equivalent baseband response of the self-interference radio channel; the radio channel comprises the RF components, such as a 3-port circulator, and the self-interference wireless channel. $h_{\text {aux,I }}(t)$

\footnotetext{
${ }^{1}$ These impulse responses include any discrepancy between the I- and Qarm, such as length difference which causes a group delay. This is likewise applicable to those counterpart baseband filter responses in the auxiliary and receiving chains.
}

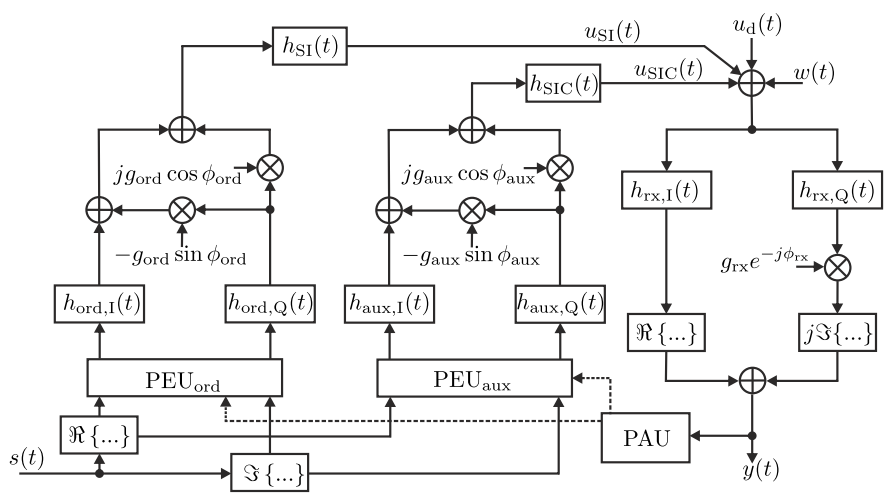

Fig. 2. Full-duplex transceiver system model. The model incorporates the $\mathrm{I} / \mathrm{Q}$ imbalances in their equivalent baseband models.

and $h_{\text {aux, }}(t)$ represent the impulse response of the baseband filters in the auxiliary transmission chain at the I- and Qarm, respectively. $h_{\mathrm{SIC}}(t)$ represents the equivalent baseband channel between the up-converting quadrature mixer at the auxiliary chain and the directional coupler at the receiver frontend.

The equivalent baseband of the RF self-interference signal at the receiving chain front-end is given by

$$
\begin{aligned}
u_{\mathrm{SI}}(t)=\mathfrak{R}\{s(t)\} & * h_{\text {ord, } \mathrm{I}}(t) * h_{\mathrm{SI}}(t)+g_{\text {ord }}\left(\mathrm{J} \cos \phi_{\text {ord }}\right. \\
& \left.-\sin \phi_{\text {ord }}\right) \mathfrak{I}\{s(t)\} * h_{\text {ord, } \mathrm{Q}}(t) * h_{\mathrm{SI}}(t),
\end{aligned}
$$

where $g_{\text {ord }}$ and $\phi_{\text {ord }}$ are respectively the gain and the phase I/Q mismatches at the ordinary chain. The equivalent baseband of the RF self-interference cancellation (SIC) signal at the receiver front-end is given by

$$
\begin{array}{r}
u_{\mathrm{SIC}}(t)=\mathfrak{R}\{s(t)\} * h_{\text {aux }, \mathrm{I}}(t) * h_{\mathrm{SIC}}(t)+g_{\text {aux }}\left(j \cos \phi_{\text {aux }}\right. \\
\left.-\sin \phi_{\text {aux }}\right) \mathfrak{I}\{s(t)\} * h_{\text {aux }, \mathrm{Q}}(t) * h_{\mathrm{SIC}}(t),
\end{array}
$$

where $g_{\text {aux }}$ and $\phi_{\text {aux }}$ are the gain and the phase I/Q mismatches at the auxiliary chain, respectively.

Ideally speaking, the self-interference signal $u_{\mathrm{SI}}(t)$ and the self-interference cancellation one $u_{\text {SIC }}(t)$ have to be exactly identical in order to assure complete cancellation of the selfinterference. Therefore, the I/Q imbalance parameters, in addition to the self-interference radio channel, have to be estimated in order to compensate their effect digitally, and reconstruct a self-interference cancellation signal that perfectly matches the interference signal, of course with negative magnitude.

The direct-down-converter structure of the quadrature demodulator is modeled in its equivalent baseband form [13], see the right block in Fig. 2. $h_{\mathrm{rx}, \mathrm{I}}(t)$ and $h_{\mathrm{rx}, \mathrm{Q}}(t)$ represent the impulse response of the LPF baseband filters at the I- and Qarm, respectively. The I/Q gain and the phase mismatches are denoted by $g_{\mathrm{rx}}$ and $\phi_{\mathrm{rx}}$, respectively. The I/Q imbalance of the receiving chain reduces the efficiency of the estimation, hence, leads us to inaccurate parameter estimation of the transmission chains' I/Q imbalances.

\section{AdVANCED PRE-EQualization Units}

The basic model of linear pre-equalization [9] $G_{\mathrm{L}}(f)=-\frac{H_{\text {ord, } \mathrm{I}}(f) H_{\mathrm{SI}}(f)}{H_{\text {aux I }}(f) H_{\mathrm{II}}(f)}$ is not able to cope with $\mathrm{I} / \mathrm{Q}$ imbalance in the full-duplex transceiver. This basic linear model assumes a perfect match between the I- and Qarm in all transceiver chains, i.e., $h_{\text {ord,I }}(t)=h_{\text {ord, }}(t)$, 
$h_{\text {aux }, \mathrm{I}}(t)=h_{\text {aux }, \mathrm{Q}}(t)$ and $h_{\mathrm{rx}, \mathrm{I}}(t)=h_{\mathrm{rx}, \mathrm{Q}}(t)$. Furthermore, the model assumes that the LO signals are perfectly shifted by $90^{\circ}$ and have an equal amplitude, thereby $\phi_{\text {ord }}=\phi_{\text {aux }}=\phi_{\mathrm{rx}}=0$ and $g_{\text {ord }}=g_{\text {aux }}=g_{\text {rx }}=1$. In real-world transceivers, however, the I/Q imbalance is an unavoidable impairment. Therefore, the linear model has a poor performance due to its limited capability in capturing a comprehensive signal form. The channel estimations are corrupted by the I/Q mismatch, consequently, the estimated pre-equalization function $\tilde{G}_{\mathrm{L}}(f)=-\frac{\tilde{H}_{\text {ord, } \mathrm{I}}(f) \tilde{H}_{\mathrm{SI}}(f)}{\tilde{H}_{\text {aux }, \mathrm{I}}(f) \tilde{H}_{\mathrm{SIC}}(f)}+\epsilon$ is deviated from its ideal value. Additionally, the deficiency of the model in calibrating the I/Q imbalance digitally is another disadvantage due to the lack of I/Q imbalance parameters estimation. A couple of advantages remain in favor of this linear model such as simplicity, moreover, a shorter pilot signal is required for estimation purpose in comparison to the other advanced models as will be explained later on.

\section{A. Frequency-independent I/Q imbalance replicator}

Fig. 3 depicts the structure of the frequency-independent I/Q imbalance (FIIQ) replicator. The FIIQ replicator is an advanced linear model able to cope with the I/Q imbalance in its frequency-independent form at both transmission chains. This replicator structure is divided virtually, based on the assigned task, into three major parts: The part which comprises the multipliers $c_{\text {aux }, \alpha}$ and $c_{\text {aux }, \beta}$ is dedicated to compensate the auxiliary chain frequency-independent I/Q imbalance, the middle part $g(t)$ contains the self-interference radio channel and the SIC one, and the last part at the left side in Fig. 3 with the multipliers $r_{\text {ord, } \alpha}$ and $r_{\text {ord, } \beta}$ is used to replicate the I/Q mismatch of the ordinary chain. The FIIQ replicator is loaded into the pre-equalization unit $\mathrm{PEU}_{\mathrm{aux}}$ as in Fig. 2, meanwhile the ordinary pre-equalizer $\mathrm{PEU}_{\text {ord }}$ remains empty unoccupied, since it is utilized by a different DSP block.

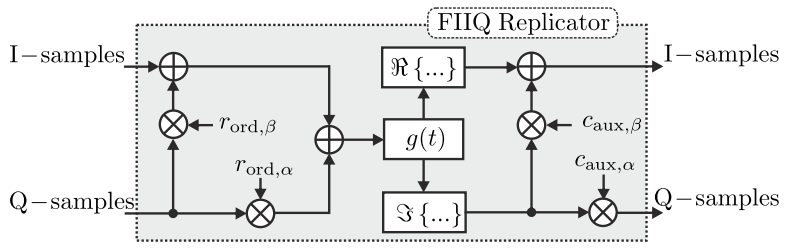

Fig. 3. The structure of the frequency-independent I/Q imbalance (FIIQ) replicator.

The frequency domain representation of the FIIQ replicator parameters are given by

$$
\begin{aligned}
G(f) & =-\frac{H_{\text {ord,I }}(f) H_{\mathrm{SI}}(f)}{H_{\text {aux }, \mathrm{I}}(f) H_{\mathrm{SIC}}(f)}, \\
r_{\text {ord }, \alpha} & =J g_{\text {ord }} \cos \phi_{\text {ord }}, \\
c_{\text {aux }, \alpha} & =\frac{1}{g_{\text {aux }} \cos \phi_{\text {aux }}}, \\
r_{\text {ord }, \beta} & =-g_{\text {ord }} \sin \phi_{\text {ord }}, \\
c_{\text {aux }, \beta} & =\tan \phi_{\text {aux }} .
\end{aligned}
$$

Note that $g(t)=\mathcal{F}^{-1}\{G(f)\}$ is an FIR filter with time domain complex coefficients and the rest of the FIIQ parameters are scalar multipliers.

\section{B. Frequency-selective $I / Q$ imbalance replicator}

Fig. 4 depicts the structure of the frequency-selective I/Q imbalance (FSIQ) replicator. Taking into account the frequency-selective behavior of the I/Q imbalance allows a better estimation of the self-interference and self-interference cancellation radio channels by mitigating the influence of the I/Q mismatch on the estimation procedure. In fact, the I/Q imbalance parameters are also estimated and integrated into the FSIQ replicator structure. The FSIQ replicator is designed to compensate the I/Q imbalance, which occurs at the auxiliary chain, and replicate the I/Q imbalance of the ordinary chain. By that, the FSIQ replicator prepares an RF self-interference cancellation signal that matches the RF self-interference signal, for which the multipath self-interference radio channel and the I/Q imbalance in its both models, the frequency-independent and frequency-selective one, are considered. Similar to the FIIQ case, the FSIQ replicator occupies the $\mathrm{PEU}_{\mathrm{aux}}$, whereas the $\mathrm{PEU}_{\text {ord }}$ is kept empty unoccupied for different purposes.

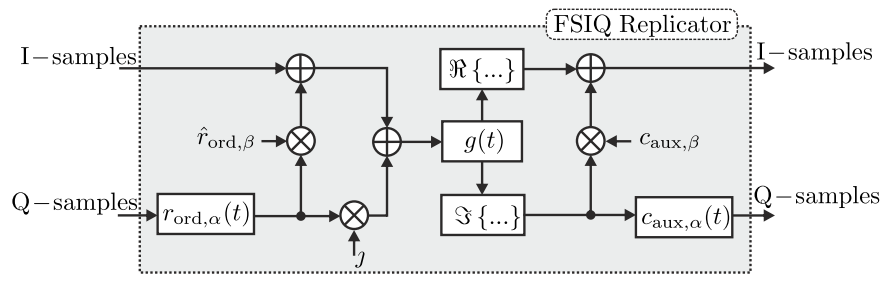

Fig. 4. The structure of the frequency-selective I/Q imbalance (FSIQ) replicator.

The FSIQ replicator parameters, in their frequency domain representation, can be expressed as

$$
\begin{aligned}
G(f) & =-\frac{H_{\text {ord,I }}(f) H_{\mathrm{SI}}(f)}{H_{\mathrm{aux}, \mathrm{I}}(f) H_{\mathrm{SIC}}(f)}, \\
R_{\text {ord }, \alpha}(f) & =\frac{H_{\text {ord,Q }}(f)}{H_{\text {ord, I }}(f)} g_{\text {ord }} \cos \phi_{\text {ord }}, \\
C_{\text {aux }, \alpha}(f) & =\frac{H_{\text {aux }, \mathrm{I}}(f)}{H_{\text {aux }, \mathrm{Q}}(f) g_{\text {aux }} \cos \phi_{\text {aux }}}, \\
\hat{r}_{\text {ord }, \beta} & =-\tan \phi_{\text {ord }}, \\
c_{\text {aux }, \beta} & =\tan \phi_{\text {aux }} .
\end{aligned}
$$

Note that $g(t)=\mathcal{F}^{-1}\{G(f)\}$ is an FIR filter with time domain complex coefficients, $r_{\text {ord, } \alpha}(t)=\mathcal{F}^{-1}\left\{R_{\text {ord, } \alpha}(f)\right\}$ and $c_{\text {aux }, \alpha}(t)=\mathcal{F}^{-1}\left\{C_{\text {aux }, \alpha}(f)\right\}$ are FIR filters with time domain real coefficients, and the rest of the FSIQ parameters $\hat{r}_{\text {ord, } \beta}$ and $c_{\mathrm{aux}, \beta}$ are scaler multipliers.

\section{Distributed I/Q imbalance compensation units}

Unlike the FIIQ and FSIQ replication models, the distributed model requires both pre-equalization units to be activated. The $\mathrm{PEU}_{\text {ord }}$ is loaded with the structure as in Fig. 5a, this PEU compensates the I/Q imbalance which occurs at the ordinary chain.

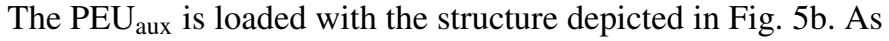
long as the I/Q imbalance at the ordinary chain is completely compensated, there is no need anymore to replicate its behavior at the auxiliary chain. In accordance to this, the $\mathrm{PEU}_{\mathrm{aux}}$ is simplified to the aforementioned structure, which is designed to compensate the auxiliary chain I/Q imbalance and preequalize the baseband signal with the self-interference and SIC radio channels. Despite of the full-duplex application, the main advantage of the distributed model is that the I/Q imbalance of the ordinary transmission signal is digitally corrected, which is desirable for the remote reception node. The distributive property of this model might be considered as disadvantage in 
some practical implementations where the access to the digital domain of the ordinary chain is not recommended or maybe not possible.

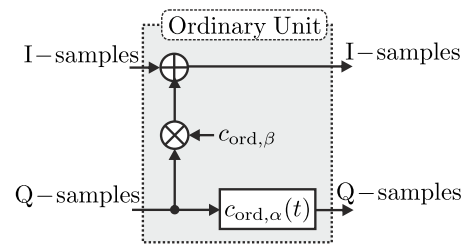

(a)

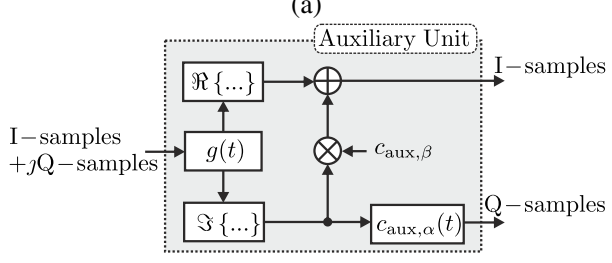

(b)

Fig. 5. The distributed I/Q imbalance compensation units; (a) The structure of the ordinary chain pre-equalization unit, (b) The structure of the auxiliary chain pre-equalization unit.

The same parameters which are used in the FSIQ replicator, set of parameters (4), are used for the distributed model except of $(4 d)$ and $(4 b)$; these parameters are replaced by $c_{\text {ord }, \beta}=\tan \phi_{\text {ord }}$ and $C_{\text {ord, } \alpha}(f)=\frac{H_{\text {ord, },}(f)}{H_{\text {ord, }}(f) g_{\text {ord }} \cos \phi_{\text {ord }}}$, respectively.

\section{SElf-INTERFERENCE WirELESS CHANNEL AND I/Q IMBALANCE PARAMETER ESTIMATION}

\section{A. Pilot signal structure and parameters}

The pilot signal is an OFDM signal with null-subcarriers for both DC and edge subcarriers [14], and with pilot subcarriers consisting of a modified Frank-Zadoff-Chu (FZC) sequence [15]. As shown in Fig. 6d, in the time domain the pilot signal is concatenated of two segments which have the following properties. The first segment has a conjugate symmetric (CS) property in its frequency domain representation, i.e., $S_{\mathrm{p}, \mathrm{cs}}(f)=S_{\mathrm{p}, \mathrm{cs}}^{*}(-f)$ [16], see Fig. $6 \mathrm{~b}$. Taking the inverse Fourier transform of this CS spectrum $s_{\mathrm{p}, \mathrm{re}}(t)=\mathcal{F}^{-1}\left\{S_{\mathrm{p}, \mathrm{cs}}(f)\right\}$ produces a pure real signal in the time domain. In contrast to the first segment, the second segment has a conjugate anti-symmetric (CA) property in its frequency representation, i.e., $S_{\mathrm{p}, \mathrm{ca}}(f)=-S_{\mathrm{p}, \mathrm{ca}}^{*}(-f)$ [16], see Fig. 6c. The time domain signal of the second segment is likewise generated by taking the inverse Fourier transform of this CA spectrum $s_{\mathrm{p}, \mathrm{im}}(t)=\mathcal{F}^{-1}\left\{S_{\mathrm{p}, \mathrm{ca}}(f)\right\}$ which produces a pure imaginary signal.

The continuous frequency domain mathematical representation of the pilot signal segments is then given by

$$
\begin{aligned}
& S_{\mathrm{p}, \mathrm{cs}}(f)=\mathfrak{R}\left\{S_{\mathrm{p}}(f)\right\}-\jmath \operatorname{sgn}(f) \mathfrak{J}\left\{S_{\mathrm{p}}(f)\right\}, \\
& S_{\mathrm{p}, \mathrm{ca}}(f)=\jmath S_{\mathrm{p}, \mathrm{cs}}(f),
\end{aligned}
$$

where $S_{\mathrm{p}}(f)$ is the spectrum of the FZC sequence, see Fig. $6 \mathrm{a}$.

The aim of such pilot structure is to improve the channel estimation by mitigating the influence of the I/Q mismatches. It further allows to estimate the I/Q imbalance out of the two segments of the pilot signal. The parameters of the pilot signal are summarized in Table I. Up so far and throughout the rest of this paper, for notational convenience, continuous time $t$ and

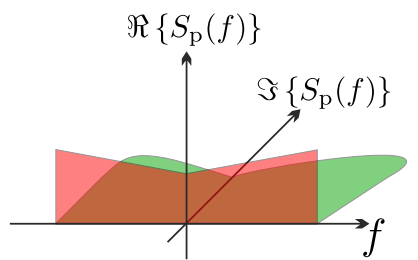

(a)

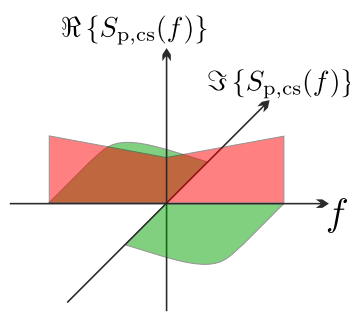

(b)

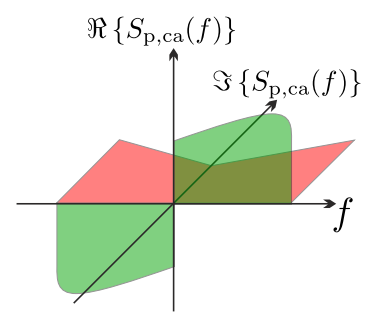

(c)

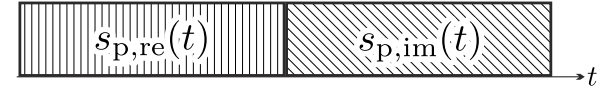

(d)

Fig. 6. Frequency and time representation of the pilot signal structure; (a) Fanciful spectrum of unmodified FZC sequence, (b) The spectrum of the pilot signal first segment, (c) The spectrum of the pilot signal second segment, (d) The time domain concatenated structure of the whole pilot signal.

frequency $f$ notations are used, even for the digital discrete signals and frequency baseband subcarriers, respectively.

TABLE I. THE OFDM PILOT SIGNAL PARAMETERS

\begin{tabular}{c|c}
\hline Parameter & Value and Unit \\
\hline \hline Number of subcarriers & 512 \\
\hline Number of null subcarriers & 96 \\
\hline Number of active subcarriers & 416 \\
\hline Number of repetitions & 10 times \\
\hline Cyclic prefix (CP) length & 16 samples \\
\hline
\end{tabular}

\section{B. Estimation procedure}

The pilot signal is consecutively sent through the ordinary and auxiliary chains to be received at the local receiver. The parameter acquisition unit (PAU) estimates the required parameters based on the implemented model of pre-equalization. Within the estimation phase, a perfect timing and frequency synchronization is assumed. It is a rational assumption due to the proximity between $\mathrm{Tx}$ and $\mathrm{Rx}$, which is resulting in a high SNR of the received pilot signal. The prefect timing property allows us to precisely split the estimated pilot into two consecutive segments. The estimated frequency responses of the ordinary chain are given by ${ }^{2}$

$$
\begin{array}{r}
Y_{\text {ord }, \alpha}(f)=S_{\mathrm{p}, \mathrm{cs}}(f) H_{\mathrm{ord}, \mathrm{I}}(f) H_{\mathrm{SI}}(f) H_{\mathrm{rx}, \mathrm{I}}(f), \\
Y_{\mathrm{ord}, \beta}(f)=g_{\text {ord }}\left(j \cos \phi_{\text {ord }}-\sin \phi_{\text {ord }}\right) S_{\mathrm{p}, \mathrm{ca}}(f) \\
\cdot H_{\mathrm{ord}, \mathrm{Q}}(f) H_{\mathrm{SI}}(f) H_{\mathrm{rX}, \mathrm{I}}(f) .
\end{array}
$$

The estimated frequency responses of the auxiliary chain are given by

$$
\begin{array}{r}
Y_{\mathrm{aux}, \alpha}(f)=S_{\mathrm{p}, \mathrm{cs}}(f) H_{\mathrm{aux}, \mathrm{I}}(f) H_{\mathrm{SIC}}(f) H_{\mathrm{rx}, \mathrm{I}}(f), \\
Y_{\mathrm{aux}, \beta}(f)=g_{\mathrm{aux}}\left(\mathrm{cos} \phi_{\mathrm{aux}}-\sin \phi_{\mathrm{aux}}\right) S_{\mathrm{p}, \mathrm{ca}}(f) \\
\cdot H_{\mathrm{aux}, \mathrm{Q}}(f) H_{\mathrm{SIC}}(f) H_{\mathrm{rx}, \mathrm{I}}(f) .
\end{array}
$$

\footnotetext{
${ }^{2}$ The additive white Gaussian noise (AWGN) at the receiver, indicted in Fig. 2 as $w(t)$, is omitted to ease the notations.
} 
Note that the receiver chain is assumed to be ideal and does not suffer from any I/Q mismatch, i.e., $h_{\mathrm{rx}, \mathrm{I}}(t)=h_{\mathrm{rx}, \mathrm{Q}}(t), g_{\mathrm{rx}}=1$ and $\phi_{\mathrm{rx}}=0$. Such assumption is practically applicable by means of digital-down-convertion [17] with higher sampling rate of the analog-to-digital converter (ADC) at the receiving chain.

Using (6), (7), (8) and (9) with the perfect knowledge of the pilot baseband (5), four estimated frequency responses can be found to be given by

$$
\begin{aligned}
& H_{\mathrm{ord}, \alpha}(f)=\frac{Y_{\text {ord }, \alpha}(f)}{S_{\mathrm{p}, \mathrm{cs}}(f)}, \quad H_{\mathrm{ord}, \beta}(f)=\frac{Y_{\mathrm{ord}, \beta}(f)}{S_{\mathrm{p}, \mathrm{ca}}(f)}, \\
& H_{\mathrm{aux}, \alpha}(f)=\frac{Y_{\mathrm{aux}, \alpha}(f)}{S_{\mathrm{p}, \mathrm{cs}}(f)}, \quad H_{\mathrm{aux}, \beta}(f)=\frac{Y_{\mathrm{aux}, \beta}(f)}{S_{\mathrm{p}, \mathrm{ca}}(f)} \text {. }
\end{aligned}
$$

These estimated responses are exploited to compute the PEU parameters. The first parameter, which is broadly used in each PEU, can be computed as follows

$$
G(f)=-\frac{H_{\mathrm{ord}, \alpha}(f)}{H_{\mathrm{ord}, \beta}(f)} .
$$

The CS frequency responses are similarly computed from the estimated responses to be given by

$$
\begin{aligned}
& C_{\text {ord }, \alpha}(f)=\frac{1}{R_{\text {ord }, \alpha}(f)}=\frac{H_{\text {ord }, \beta}(f)}{H_{\text {ord }, \alpha}(f)}+\frac{H_{\text {ord }, \beta}^{*}(-f)}{H_{\text {ord }, \alpha}^{*}(-f)}, \\
& C_{\text {aux }, \alpha}(f)=\frac{H_{\mathrm{aux}, \beta}(f)}{H_{\text {aux }, \alpha}(f)}+\frac{H_{\mathrm{aux}, \beta}^{*}(-f)}{H_{\mathrm{aux}, \alpha}^{*}(-f)} .
\end{aligned}
$$

The phase mismatches of the ordinary and auxiliary chain are computed by averaging over the frequency to be given by

$$
\begin{aligned}
& \phi_{\mathrm{ord}}=\arctan \left(-\frac{J}{B} \int_{-B / 2}^{+B / 2} \frac{\frac{H_{\mathrm{ord}, \beta}(f)}{H_{\mathrm{ord}, \alpha}(f)}-\frac{H_{\mathrm{ord}, \beta}^{*}(-f)}{H_{\mathrm{ord}, \alpha}^{*}(-f)}}{\frac{H_{\mathrm{ord}, \beta}(f)}{H_{\mathrm{ord}, \alpha}(f)}+\frac{H_{\mathrm{ord}, \beta}^{*}(-f)}{H_{\mathrm{ord}, \alpha}^{*}(-f)}} \mathrm{d} f\right), \\
& \phi_{\text {aux }}=\arctan \left(-\frac{J}{B} \int_{-B / 2}^{+B / 2} \frac{\frac{H_{\mathrm{aux}, \beta}(f)}{H_{\mathrm{aux}, \alpha}(f)}-\frac{H_{\mathrm{aux}, \beta}^{*}(-f)}{H_{\mathrm{au}, \alpha}^{*}(-f)}}{\frac{H_{\mathrm{aux}, \beta}(f)}{H_{\mathrm{aux}, \alpha}(f)}+\frac{H_{\mathrm{aux}, \beta}^{*}(-f)}{H_{\mathrm{aux}, \alpha}^{*}(-f)}} \mathrm{d} f\right) \text {, }
\end{aligned}
$$

where $B$ is the bandwidth of the pilot signal.

For practical implementation by means of FIR filters, the filter coefficients can be computed by taking the DFT of the pilot signal subcarriers with maximum length equal to the pilot signal cyclic prefix.

\section{A. Simulation parameters}

\section{Simulation}

A simulation framework has been built to simulate the behavior of the full-duplex transceiver chains. The PEU models have been tested under realistic system behavior. The LO parameters concerning the frequency-independent I/Q imbalance are listed in Table II. The frequency-selective I/Q mismatches are simulated by means of FIR filters of order 9.

TABLE II. FI-I/Q IMBALANCE SIMULATION PARAMETERS

\begin{tabular}{c||c|c|c} 
& Ordinary & Auxiliary & Receiving \\
\hline Gain mismatch $g$ & $0.7485 / \mathrm{dB}$ & $0.5877 / \mathrm{dB}$ & $0.2996 / \mathrm{dB}$ \\
\hline Phase mismatch $\phi$ & $5.5^{\circ}$ & $2.7^{\circ}$ & $2^{\circ}$
\end{tabular}

\section{B. Simulation results}

The influence of the frequency-independent I/Q imbalance over the self-interference suppression amount has been analyzed. Fig. 7 illustrates the gain and phase mismatches versus the achieved amount of self-interference suppression. The ordinary chain is kept ideally operating without any I/Q imbalance, whereas the I/Q imbalance in the auxiliary chain is gradually increased in order to report the results of the linear PEU under such conditions.

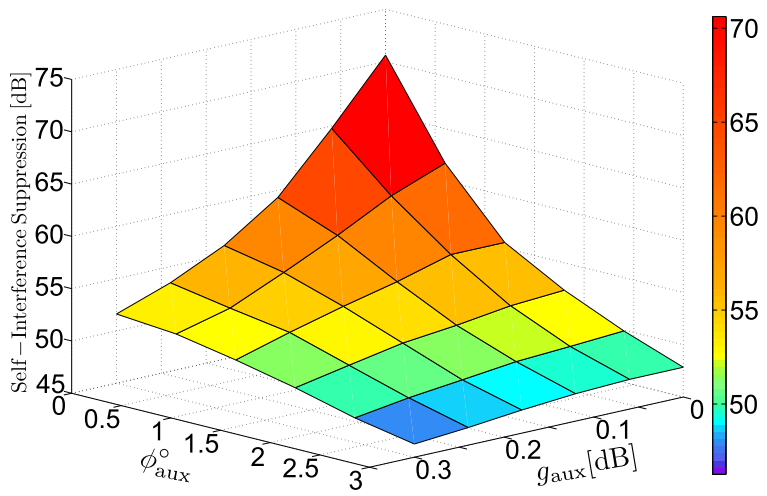

Fig. 7. Self-interference suppression performance deterioration due to increasing number of the frequency-independent I/Q mismatch between the two transmission chains

The linear PEU is compared to the FIIQ replicator under I/Q mismatch transceiver behavior in its frequency-independent model. The considered I/Q imbalance parameters are listed in Table II. Two configurations are simulated, and their results are documented in Table III. In the first configuration an ideal receiving chain is considered, while in the second configuration a receiving chain corrupted with I/Q imbalance is simulated.

TABLE III. THE ACHIEVED SELF-INTERFERENCE SUPPRESSION UNDER FI-I/Q IMBALANCE BEHAVIOR

\begin{tabular}{|c|c|c|}
\cline { 2 - 3 } \multicolumn{1}{c||}{} & Rx-I/Q disabled & Rx-I/Q enabled \\
\hline Linear & $47.2 \mathrm{~dB}$ & $47.1 \mathrm{~dB}$ \\
\hline FIIQ replicator & $71.8 \mathrm{~dB}$ & $61.6 \mathrm{~dB}$ \\
\hline
\end{tabular}

Finally, a highly realistic model of I/Q imbalance is simulated. The model incorporates the I/Q imbalance in its both models, the frequency-independent and the frequency-selective one, for all transceiver chains. All proposed PEUs are tested under two configuration: with and without receiver I/Q imbalance. The simulation results are listed in Table IV.

TABLE IV. THE ACHIEVED SELF-INTERFERENCE SUPPRESSION UNDER FS-I/Q IMBALANCE BEHAVIOR

\begin{tabular}{|c||c|c|}
\cline { 2 - 3 } \multicolumn{1}{c||}{} & Rx-I/Q disabled & Rx-I/Q enabled \\
\hline Linear & $46.2 \mathrm{~dB}$ & $45.7 \mathrm{~dB}$ \\
\hline FIIQ replicator & $51.3 \mathrm{~dB}$ & $51.1 \mathrm{~dB}$ \\
\hline FISQ replicator & $71 \mathrm{~dB}$ & $56.6 \mathrm{~dB}$ \\
\hline Distributed PEU & $70.5 \mathrm{~dB}$ & $57.3 \mathrm{~dB}$ \\
\hline
\end{tabular}

\section{Performance evaluation}

Fig. 7 shows the deterioration in the linear PEU performance as the I/Q mismatch between the ordinary chain and the auxiliary one is increased. This demonstrates the significance of the advanced I/Q pre-equalization models. 
As the results in Table III indicate, the FIIQ replicator outperforms the linear PEU under frequency-independent I/Q imbalance transceiver behavior. The receiver I/Q imbalance disturbs the estimation phase and consequently decreases the accuracy of the estimated parameters. Hence, the FIIQ replicator has a better performance when the Rx I/Q is disabled.

The results in Table IV show a significant deterioration in the FIIQ replicator performance when a frequency-selective model of I/Q imbalance is used. The FIIQ replicator demonstrates a relatively poor performance even with an ideal implementation of the receiving chain. The FSIQ replicator has approximately similar results to the distributed PAU. Both the FSIQ replicator and the distributed PAU demonstrate the best performance in suppressing the self-interference even with a highly realistic model of the I/Q imbalance, i.e., frequency-independent and frequency-selective. The I/Q imbalance of the receiving chain has a huge negative influence on these PAUs.

\section{CONCLUSIONS}

Three advanced pre-equalization units (PEUs) have been discussed in this work. The FIIQ replicator has shown a higher level of self-interference suppression in comparison to the basic linear model. The FSIQ replicator and the distributed PEU have shown the best performance in suppressing the selfinterference. Nevertheless, the FIIQ replicator is still an option where the complexity of the design is matter as the available computational resources are quite limited. The FSIQ replicator and the distributed PEU are pretty similar with respect to their complexity and performance, but they are different in structure. For implementation purpose, the FSIQ replicator might be a good choice when a conventional half-duplex transceiver is needed to be upgraded in order to operate in full-duplex scheme without any additional tweaks in the ordinary chain digital domain. The distributed PEU is recommended to be implemented as the I/Q imbalance in the ordinary chain is corrected. This correction is at the expense of dual digital domain access at both transmission chains.

This work focused on the influence of I/Q imbalance for full-duplex systems, while the impact of nonlinear distortions, as they are mainly stemming from the power amplifier, has been neglected. The importance of considering the nonlinearity in full-duplex systems has been investigated in [18], and first nonlinear digital self-interference cancellation techniques have been reported in [4], [9], [19], [20]. In future work the proposed model will be extended to also include these nonlinear impairments, beside the impairments due to $\mathrm{I} / \mathrm{Q}$ imbalance. The concept of joint modeling of nonlinear distortion and I/Q imbalance has been introduced in [21] and extended to a Volterra series based approach in [22]. This model, however, captures only the case of transmitter-side predistortion and might have to be adapted for the full-duplex scenario.

\section{REFERENCES}

[1] J. I. Choi, M. Jain, K. Srinivasan, P. Levis, and S. Katti, "Achieving single channel, full duplex wireless communication," in Proceedings of the sixteenth annual international conference on Mobile computing and networking, ser. MobiCom '10, 2010, pp. 1-12.

[2] M. A. Khojastepour, K. Sundaresan, S. Rangarajan, X. Zhang, and S. Barghi, "The Case for Antenna Cancellation for Scalable Full-duplex Wireless Communications," in Proceedings of the 10th ACM Workshop on Hot Topics in Networks, ser. HotNets-X, 2011, pp. 17:1-17:6.

[3] M. Duarte and A. Sabharwal, "Full-duplex wireless communications using off-the-shelf radios: Feasibility and first results," in Signals, Systems and Computers (ASILOMAR), 2010 Conference Record of the Forty Fourth Asilomar Conference on, 2010, pp. 1558-1562.
[4] D. Bharadia, E. McMilin, and S. Katti, "Full duplex radios," in Proceedings of the ACM SIGCOMM 2013 conference on SIGCOMM, ser. SIGCOMM '13, 2013, pp. 375-386.

[5] D. Bharadia and S. Katti, "Full Duplex MIMO Radios," in 11th USENIX Symposium on Networked Systems Design and Implementation (NSDI 14), Apr. 2014, pp. 359-372.

[6] E. Everett, A. Sahai, and A. Sabharwal, "Passive Self-Interference Suppression for Full-Duplex Infrastructure Nodes," Wireless Communications, IEEE Transactions on, vol. PP, no. 99, pp. 1-15, 2014.

[7] M. Jain, J. I. Choi, T. Kim, D. Bharadia, S. Seth, K. Srinivasan, P. Levis, S. Katti, and P. Sinha, "Practical, real-time, full duplex wireless," in Proceedings of the 17th annual international conference on Mobile computing and networking, ser. MobiCom '11, 2011, pp. 301-312.

[8] M. Duarte, C. Dick, and A. Sabharwal, "Experiment-Driven Characterization of Full-Duplex Wireless Systems," Wireless Communications, IEEE Transactions on, vol. 11, no. 12, pp. 4296-4307, December 2012.

[9] R. Askar, T. Kaiser, B. Schubert, T. Haustein, and W. Keusgen, "Active self-interference cancellation mechanism for full-duplex wireless transceivers," in Cognitive Radio Oriented Wireless Networks and Communications (CROWNCOM), 2014 9th International Conference on, June 2014, pp. 539-544.

[10] D. Korpi, L. Anttila, V. Syrjälä, and M. Valkama, "Widely Linear Digital Self-Interference Cancellation in Direct-Conversion Full-Duplex Transceiver," Selected Areas in Communications, IEEE Journal on, vol. 32, no. 9, pp. 1674-1687, Sept 2014.

[11] A. Sahai, G. Patel, C. Dick, and A. Sabharwal, "On the Impact of Phase Noise on Active Cancelation in Wireless Full-Duplex," Vehicular Technology, IEEE Transactions on, vol. 62, no. 9, pp. 4494-4510, Nov 2013.

[12] J. Luo, A. Kortke, and W. Keusgen, "Joint calibration of frequency selective time variant I/Q-imbalance and modulator DC-offset error in broadband direct-conversion transmitters," in Communications, Circuits and Systems, 2009. ICCCAS 2009. International Conference on, July 2009, pp. 255-259.

[13] P. Rykaczewski, J. Brakensiek, and F. Jondral, "Towards an analytical model of I/Q imbalance in OFDM based direct conversion receivers," in Vehicular Technology Conference, 2004. VTC 2004-Spring. 2004 IEEE 59th, vol. 4, May 2004, pp. 1831-1835 Vol.4.

[14] J. Luo, A. Kortke, and W. Keusgen, "Efficient Channel Estimation Schemes for MIMO OFDM Systems with NULL Subcarriers," in Vehicular Technology Conference, 2008. VTC 2008-Fall. IEEE 68th, 2008, pp. 1-5.

[15] D. Chu, "Polyphase codes with good periodic correlation properties (Corresp.)," IEEE Transactions on Information Theory, vol. 18, no. 4, pp. 531-532, 1972.

[16] S. K. Mitra, Digital Signal Processing: A Computer-Based Approach, 2nd ed. McGraw-Hill, 2001.

[17] T. Hollis and R. Weir, "The Theory of Digital Down Conversion," Hunt Engineering, Tech. report v1.2, 2003.

[18] D. Korpi, T. Riihonen, V. Syrjälä, L. Anttila, M. Valkama, and R. Wichman, "Full-Duplex Transceiver System Calculations: Analysis of ADC and Linearity Challenges," Wireless Communications, IEEE Transactions on, vol. PP, no. 99, pp. 1-1, 2014.

[19] L. Anttila, D. Korpi, V. Syrjälä, and M. Valkama, "Cancellation of power amplifier induced nonlinear self-interference in full-duplex transceivers," in Signals, Systems and Computers, 2013 Asilomar Conference on, Nov 2013, pp. 1193-1198.

[20] E. Ahmed, A. Eltawil, and A. Sabharwal, "Self-interference cancellation with nonlinear distortion suppression for full-duplex systems," in Signals, Systems and Computers, 2013 Asilomar Conference on, Nov 2013, pp. 1199-1203.

[21] L. Anttila, P. Händel, and M. Valkama, "Joint Mitigation of Power Amplifier and I/Q Modulator Impairments in Broadband Direct-Conversion Transmitters," Microwave Theory and Techniques, IEEE Transactions on, vol. 58, no. 4, pp. 730-739, April 2010.

[22] B. Schubert, A. Gökceoglu, L. Anttila, and M. Valkama, "Augmented Volterra predistortion for the joint mitigation of power amplifier and I/Q modulator impairments in wideband flexible radio," in Global Conference on Signal and Information Processing (GlobalSIP), 2013 IEEE, Dec 2013, pp. 1162-1165. 